\title{
Some artificial intelligent techniques to design robotic systems
}

\author{
George L. Kovács \\ CIM Research Laboratory, Computer and Automation Research \\ Institute, Hungarian Academy of Sciences, \\ Budapest, Hungary, 1111 Kende u. 13-17. \\ Phone: 36-1-209-6143, Fax: 36-1-1667-503, e-mail: \\ h673kov@ella.hu,gyorgy.kovacs@sztaki.hu
}

\begin{abstract}
All types of automated manufacturing systems, which are often balanced automation systems are broadly using robots, manipulators, etc. This paper gives a restricted survey on the application of some of the so called artificial intelligence (AI) methods and tools which can be used to model, simulate, design and implement systems with robot and manipulator applications. AI in design and planning of robotic systems means the application of Multi-Agent Systems (MAS), Expert Systems (ES), Knowledge Based Systems (KBS), Artificial Neural Networks (ANN), Petri nets, Fuzzy Systems (FS), Genetic Algorithms (GA), Object-Oriented Technologies (OO), and several other things including pattern recognition, vision, voice and tactile systems and learning. Modelling and simulation in our recent understanding are more design assistance tools, than the goal of AI applications thus they completely fit into our recent topics. The paper deals not only with manufacturing and assembly applications, but with other intelligent robotic issues as well, as autonomous robots, moving robots, surgery robots, etc.
\end{abstract}

\section{Keywords}

Artificial intelligence methods, robotic systems, system design, planning

\footnotetext{
The original version of this chapter was revised: The copyright line was incorrect. This has been corrected. The Erratum to this chapter is available at DOI: 10.1007/978-0-387-35390-6_58 


\section{INTRODUCTION}

A robotic system may mean everything from a simple, single manipulator to totally equipped robotized CIM systems, however single manipulators and robots, systems of two or more robots, Flexible Manufacturing and Flexible Assembly Systems (FMS, FAS) with service robots are the typical examples. Even specific robotic applications as micro-robots, surgery robots, space and underwater robots, walking and wall climbing robots, etc. should be taken into account .

Design (and/or planning) means only some steps in the life-cycle of a robot or robotic system. The main phases of a life-cycle are: requirements specification, analysis, design, implementation, test, operation, maintenance, dismounting, reuse and recycling of the system or its parts. Design has to take into account all previous and later phases of the life-cycle as well. Each of these steps consists of several different components which may differ in the case of different products/systems. For instance design may be split into conceptual design, preliminary design, detailed design, etc. Simulation - what was not mentioned separately - is generally important in the design and in the operation phase as well, however simulation of other steps may be useful, too.

$\mathrm{AI}$ in design and planning of robotic systems means the application of MultiAgent Systems (MAS), Expert Systems (ES), Knowledge Based Systems (KBS), Artificial Neural Networks (ANN), Petri nets, Fuzzy Systems (FS), Genetic Algorithms (GA), Object-Oriented Technologies (OO), and several other things including pattern recognition, vision, voice and tactile systems and learning. These paradigms may be used as stand alone ones, or almost any combinations of 2,3 or more of them has application advantages and examples.

As a further example the paradigm of Concurrent Engineering (CE) gives further possibilities for AI applications.

All the above topics have a huge literature in journals and periodicals, such as [1-12] and in books and conference proceedings, which gives us a very diverse picture of issues we wish to tackle according to the title of the paper. However most robotics literature deals with intelligent applications as motion and path planning, collision avoidance, grasping, autonomous robots, mobile robots, walking robots, obstacle avoidance, jumping robots, navigation, humanoid robots, multi-robot systems, tele-robotics, tele-operation, tele-presence, etc., and only a small fraction deals with simulation and design issues of robotic systems. On the other hand FMS and FMC design (using robots, too) are discussed very often, having in mind the selection and layout, and integration of robots, machine tools and other equipment. Another common issue is the re-design, or re-configuration of the systems in the case of any changes, as breakdowns, extension of the system, improvement of the system, etc. 


\section{APPLICATIONS OF MODELING, SIMULATION, MULTI-AGENT AND AI METHODS AND TOOLS}

The following robotic applications are selected from the literature according to some major features of the discussed systems, however some of them would fit into other domains, too. For example simulation and design should not be always separated, etc.

\subsection{Modelling and Simulation in the Design of Robotic Systems}

Modelling, and model based simulation can be taken into account as highly effective design tools. In a robot-rich environment (environment with robots) there are many robot-specific design tasks. These belong either directly to the robots, or to the environment (as e.g. an FMS), and their co-operation in the appropriate synergy is very important As simulation examples multirobot systems, teleoperation and a robot-human motion planning system will be discussed.

M. K. Habib in [13] (Simulation Environment for an Autonomous and Decentralized Multi-Agent Robotic System, pp. 1550-1557) presents ACTRESS: „ACTor based Robot and Equipment Synthetic System”. ACTRESS is an autonomous and decentralized system consisting of robotor's. A robotor is a robot, a computing system or any equipment.

The main issue of this work is to simulate and analyse the main functions of each agent, to design and analyse the actions to be carried out by the agents, analysing real-time behaviour with a variety of situations considering their environment and the treatment of unexpected events. Path planning is solved by means of rule based, expert systems.

Based on the simulation and analysis the design of an autonomous mobile robot system is assisted. The system is taken into account as a Multi-Agent system.

Sukhan Lee and Hahk Sung Lee in [13] (Modeling, Design and Evaluation for Advanced Teleoperator Control Systems, pp. 881-888) discuss a new type of teleoperation system (telemonitoring) using simulation.

In designing and controlling teleoperation system under time delay, it is necessary to incorporate a human dynamic behaviour into the control loop. Time delay between the human operator and the remote stations may degrade the fidelity and may incur control instability. Solution is feedback signals of force, velocity and position. Another solution is and indirect control link by using a delay-free task environment. In the letter case a model and a simulated environment is needed.

Haidegger and Kopácsi in [14] present real and simulated examples for telepresence in robotics for manufacturing applications using multi-media communications.

T. Skewis, V. Lumelsky in [13] (Simulation of Sensor-Based Robot and Human Motion Planning, pp. 933-940.) claims the advantages of simulation, i.e. 
it is safer and cheaper to Simulate instead of build and test, when unknown obstacles may be in the environment of a moving robot. Modelling various robots, sensor systems, obstacles, testing several 2D and 3D motion planning algorithms, testing human performance in motion planning, plus real time animation are the main issues of this paper.

Path planning with complete information is a typical off line activity, while path planning with incomplete information or sensor-based motion planning is a real-time task.

Together with the motion problems the following main sensory types are simulated and discussed: tactile sensing, proximity sensing, visual sensing, sensing for the test of human motion planning.

\subsection{Design and Integration of Robotic Systems}

A rather good summary of design and integration issues can be found in an early reference, in [15] (Esprit Project 623 (1985-1990)). This was one of the first joint European Projects. It was published as a book to study the benefits of off-line robot programming and to study the problems of robot systems planning.

The above fields were identified and analysed in the frame of the esprit Project 75: Design Rules for the Integration of Industrial Robots into CIM Systems. Not only design, but integration is discussed, as recent complex systems may have very serious integration problems. The integration of robots into manufacturing cells/systems requires the integration of information concerning product design, plant availability and system layout as well.

The result of the study is a prototype planning system for robotized workcells. Integration of planning and off-line programming systems using simulation models is solved, too.

Design examples are given for automotive, electric/electronic and mechanical engineering industries, in four application areas: handling, machining, assembly and testing, as planning and programming of a dot matrix printer assembly and programming of the Cranfield assembly benchmark.

A rather general and important issue is the application of standards, where possible: IGES, STEP (product description), IRL (documentation purposes, readable, supports modularization) and ICR (cheap implementation, less memory and low speed processors, faster execution, for robot control) interfaces.

Sugato Bagchi and Kazuhiko Kawamura in [13] (An Architecture of a Distributed Object-Oriented Robotic System, pp. 711-716.) deals with blackboard based, distributed and object-oriented (OO) systems from the point of view of robot design.

Distributed and $\mathrm{OO}$ concepts are used to design and implement a single manipulator robotic system (ISAC: Intelligent SoftArm Control, to assist physically handicapped). An OO Communication framework, where inter-module communication is managed through remote method calls from client to server 
resource objects is presented. The distributed modules receive or send tasks through a task blackboard. There is a multi-agent, OO task decomposition and execution in ISAC. C++ is used in a networked UNIX environment. Distributed systems are generally for multiple robots, but here the functions, as manipulator controller, task planner, vision, other sensors are distributed among processes and processors. Distribution helps in integration of programs written by different groups, or to add new parts, etc.

G. Grübel et. al in [16] (ANDECS: A Computation Environment for RobotDynamics Design Automation, pp. 1088-1093.) describe a computational environment for robot-dynamic design.

The system has $\mathrm{OO}$ environments for mechatronics and robot dynamics modelling, for simulation of the robot-dynamics (with event-driven differentialalgebraic equations), for multiobjective parameter and trajectory optimisation, and for multivariate result visualisation. All parts of the system are coherently integrated using a common engineering database system.

Modelling is done by the OO Dymola language and by the DSblock system developed for the ANDECS project. The run time environment of ANDECS is DSSIM with three unique features:

- close coupling with engineering database

- powerful signal generator

- design by optimisation

Gairola in [17] (Design for Assembly: A Challenge for Expert Systems, pp. 199-212.) deals with one of the key issues which belong to product design, but which is present because of the application of robotic assembly systems. Application of expert systems for design for assembly (DFA) means cost saving, reducing rework, increasing quality, minimising material cost, etc., and it has the following major steps: simplify assembly task, improve assembly organisation and facilitate assembly execution.

\subsubsection{Component selection and layout design}

The component selection of robotics and manufacturing systems is one of the tasks which calls for the application of expert, or knowledge based (KB) systems, as all necessary inputs, requirements, possibilities and results are easy to define. As input generally the tasks to be done should be defined in the form of process plans or operation plans. The possibilities are defined by means of some data bases containing all available resources, and by means of KBs to define all constraints, relationships, and specific requirements. The KBs contain all applicable optimisation criteria, too, in the form of rules and facts (sometimes represented in frames). Even uncertain and uncomplete knowledge can be managed without problems. The main task of the $\mathrm{KB}$ system is then a match making between the goals to be achieved and the means available, taking into consideration all requirements and constraints, and some optimisation criteria. 
This component selection is called configuration, or design in several cases, however design contains layout planning as well, when the physical position of all equipment in a workshop is designed based on the available space and the selected components. If a new set of components is selected for a given task, or changes of an existing system are necessary, or different optimisation criteria are used, a reconfiguration, or redesign takes place.

Kopacek et al in [18] give an example (Semiatomatic Knowledge Based Planning of Small Assembly Cells, pp. 574-582.) how to select and make the layout of robots and machine tools.

The robot selection is based on the maximum number of axes, on the type of the robot, on the number of robots and on the maximum payloads. The selection of grippers, gripper changing devices and fixtures are done similarly.

Fisher and Maimon in [19] (Selection of Robots, pp. 172-187.) discuss a twophase model which is used for decision-making. First a set of specification is created to serve as functional specification for robot design based on the analysis of the technological requirements of a specific set of robot-related tasks, then the best fit robots are selected from the set of available robots.

The robot specifications contain about 25 different, important parameters as power requirements, control, accuracy, force, torque, gripper, integration possibilities, price, etc.

A mathematical programming method and a rule based expert system is presented to find the best set of robotic technologies that are required by the set of considered tasks (phase one). For the second phase the acceptable set of robots are chosen and then the candidate list is prioritised with both the above methods. Finally the combination of the mathematical tools (integer programming) and rule based expert systems are suggested.

Malmborg et al in [19] (An Expert System for Selection of Material Handling Equipment, pp. 484-504.) deals with industrial truck selection, as example. The paper discusses how to build up a knowledge base and then how to use it for selection. About 20 data, as code, basic design and shape, load capacity, maximum lift, lifting mechanism, etc. is used to produce all rules in PROLOG.

McGlennon et al. in [19] (ROBOSPEC - A Prototype Expert System for Robot Selection, pp. 505-527.) discusses a rule based, menu driven and user friendly system written in OPS5, which was one of the early, dedicated expert system writing language. The resulting robot specification includes: kinematics. axes, drives, programming, speed, etc.

Several references of the author and his team $[20,21,22,23,24,25,26,27]$ reflect some works concerning the application of expert systems for robotized manufacturing system design. The basic idea was to match the requirements with the possibilities, similarly to the works discussed above.

Kusiak and Heragu in [28] (Rule based expert systems for equipment selection and layout, pp. 153-191, and pp. 566-575) describe the KBML system containing 60 complex rules. 
The input of the system is the number of machines and robots, machine dimensions, connectivity matrix, location restrictions, type of possible layouts, type of material handling systems, floor dimensions, etc. and the solution is given for the simplest, single-row layout.

Paasiala et al. in [29] (Automatic Component Selection, pp. 303-312.) deals with another $\mathrm{KB}$ system. The main steps of the selection process are the following: creation of database, eliminate unsuitable components by calculations, calculating quality functions for the candidate components, sorting the components according to the quality factors, selection of components.

The STEP standard is used for data transfer of components to an object-oriented data base and to an expert system. The paradigm of Concurrent Engineering is used, where applicable.

\subsubsection{Robot and manipulator design}

Several aspects of robot, manipulator and robot system design will be discussed under this title. We do not go into basic design questions, rather try to point out interesting questions and views. Some general design aspects will be detailed, and then genetic algorithms, formal specifications and mathematical models, the multi-agent approach and intelligent autonomous robots will be discussed through application examples.

Putnik and Silva in [18] (Knowledge Integrated Manufacturing Systems, pp. 688-698.) propose a basic reference model for Knowledge Integrated Manufacturing Systems (KIMS). This system is rather general, and it can be used for robotic systems as well. The basic idea is to combine integration and knowledge, as it is done in CIM (Computer Integrated Manufacturing), or in IMS (Intelligent Manufacturing System)

Filho and Carvalho in [30] (An Integrated Strategic Planning System, pp. 209216.) define two stages of planning:

- A stochastic model to provide an aggregated planning and then decomposition for a detailed planning, and the two planning activities are integrated. A stochastic optimisation model and an equivalent deterministic model is given with detailed mathematics.

- The second stage is assignment. It has another well manageable mathematical model.

Gradetsky et al. in [30] (Concurrent Design Methods for Assembly System and Reliability Problems in Design of Assembly Systems, pp. 289-295.) tackles some major design issues. In this work a method is suggested, where products and robotic assembly systems are designed at the same time, optimisation and simulation steps are repeated. Optimal reliability is defined and calculated with precise mathematics. 


\subsubsection{Genetic algorithms}

Generic algorithms are a kind of imitation of how living nature works. It is interesting to realise, how effectively such algorithms can be used to solve different design tasks.

Jin-Oh Kim and Pradep K. Khosla in [13] (A Multi-Population Genetic Algorithm /MPGA/ and Its Application to Design of Manipulators, pp. 279-286.) give a detailed analysis of genetic algorithms and their application.

MPGA is an efficient optimisation technique for highly non-linear problems. It is a parallel implementation of GA, which is a kind of adaptive search method and it is robust for the manipulator design to best fit to the tasks to be solved. The optimality is based on the use of a dexterity measure, here it is the relative manipulability. This is a typical Task Based Design (TBD) task. The number of optimisation functions equals to the number of task points. A progressive design framework is added to the system. Generally the optimisation function is a sum of an objective function and of several constraints, this increases the complexity of the TBD exponentially with the increase of the number of task points, due to the huge search space and nonlinearity. The design constraints are: reachability, heuristics, joint limit, joint angle change between two adjacent task points; these are task specific.

J. Sasaki et al in [16] (Optimal Arrangement for Handling Unknown Objects by Co-operative Mobile Robots, pp. 112-117.) deals with robot arrangement (layout) to maximise the possibility of grasping an object by a group of robots without any troubles. Based on quantitative relationships between the arrangement, the load and the unknown parameters of each robot can be calculated using well manageable matrix notations, and functions. Simulations are given to prove the correctness of calculations. This paper makes a bridge towards our next topics.

\subsubsection{Multi-agent approach}

Multi-Agent Approach is used when a system is decomposed (or built up) in a way, when different, co-operating subsystems work together with data and information exchange to commonly solve problems. These may be „simple" problems, or problems when multiple objects are involved. Such a natural problem is the solution of the design and operation of multiple (autonomous, moving) robots. If the communicating agents have some intelligence, we call them intelligent agents, however even non-intelligent agents may have intelligent cooperation, giving intelligence to a whole system. On the other hand nonintelligent co-operation of intelligent agents may result in a non-intelligent system. In the last years multi-robot systems and multi-agent approach have a very rich literature, even in the aspects of design. 
D. Duhaut in [31] (Using a Multi-Agent Approach to Solve the Inverse Kinematics Problem. pp. 223-229.) deals with robot trajectory design.

S. Bussmann and Y. Demazeau in [32] (An Agent Model Combining Reactive and Cognitive Capabilities, pp. 2095-2102.) present some results concerning agents taking into account cognitive features, using $\mathrm{KB}$ systems. As application autonomous mobile robots are taken into account.

An abstract model of an autonomous agent is presented. It integrates reactive and cognitive behaviour. For such an integration the following requirements should be accomplished: reactivity, timely behaviour and symbolic representation. Knowledge representation, planning, scheduling and execution plus the process of evaluation is added. The evaluation module constantly supervises the environment and the agent's actions to ensure the agent's reactivity. The reactive agent model specifies an agent by its behaviour, i.e. how the agent reacts to certain stimuli from the environment. With every perceptual input, or class of inputs certain actions are associated, which are performed immediately. Different solutions can be used, such as programmed, hard-wired, rule-based, or multi-layered architecture. Conflicts of simultaneously firing rules is solved by priorities.

Cognitive (or intentional) agent models are motivated by the agent's knowledge. AI techniques lead to the introduction of beliefs and intentions into the reasoning process of the agents. Intentions enable the agent to reason about its internal state and that of the others. In the BDI architecture [33] belief, desires and intentions form the basis of all reasoning processes.

D. Lambrinos and C. Schreier in [16] (Building Complete Autonomous Agents: A case Study on Categorisation, pp. 170-177.) report on intelligent agents. Complete agents should be built to understand intelligence. A complete agent behaves autonomously in its environment without human intermediary. A new control architecture (Extended Braitenberg Architecture) consisting of loosely coupled parallel running processes is used to control a mobile robot that collects objects and categorise them. Detailed mathematical formulations are given for navigation, exploration, learning, etc.

R. Alami in [16] (A Fleet of Autonomous and Co-operative Mobile Robots, pp. 1112-1117.) gives a generic, co-operative scheme for multi-robot co-operation based on an incremental and distributed plan-merging process. A co-operative scheme has coherence in all situations, including the avalanche which may occur after a failure. It is able to detect dead-lock situations to call for a new task distribution process, if needed. The scheme can be used in a hierarchical manner, and in a context where planning is performed parallel with plan execution (CE approach).

Friedrich, Rogalla and Dillmann in [34] (Integrating skills into multi-agent systems, pp. 357-366.) deals with Multi-Agent Robot Systems (MAS). Agents require agent-specific but flexible skills to cope with their tasks and environment's variability. Elementary Operations (EO) are presented to combine 
the agent specific nature of skills with the requirements for a general action knowledge representation inherent to MASs.

The main advantages of MASs are the modularity, robustness and fault tolerance, maintainability and extendibility. There are two models to describe MAS: the single agent model and the system model which is a collection of agents. Mathematical and set theoretical descriptions of MASs and of skills are given in details.

M. Minagawa and Y. Kakazu in [35] (Multiple Robots Navigation in Cellular Space: Acquisition of Collective Behaviour, pp. 1517-1523.) deal with autonomous robots in a 2D space. The problem is to get the robots from an initial state to a goal state. These states mean locations. During the motion the agents appear as moving obstacles to each other.

Task oriented ANN based behaviour functions with sensory inputs and motor outputs are assigned to the agents, and the problem is solved by Evolutionary Programming (a real valued mutation on network weights occurs according to Gaussian distribution). Deadlock and reactivation (with a simple energy transfer and sharing mechanism) problems are solved by local interactions between the agents. Optimal motion was not reached, as the method of conflict resolution should be refined.

\subsubsection{Intelligent autonomous robots}

Some papers from the previous session already dealt with intelligent autonomous robots, as agents, this session gives some more application examples for design.

E. Freund, J. Rossmann in [32] (Intelligent Autonomous Robots for Industrial Space Applications, pp. 1072-1080.) show how to provide high flexibility and autonomy for robot systems, together with consistent and user-transparent concepts for installation, programming and operation of robots in their working environment. They concentrate on control (CIROS: Multirobot controller) with modern man-machine interfaces for intuitive operation. The system COSIMIR (Cell Oriented Simulation of Industrial Robots) is the simulator of control (PCROB) and display of the user environment, of the robot controller, and of the environment.

J. Schweiger, K Ghandri and A. Koller in [32] (Concepts of a Distributed RealTime Knowledge Base for Teams of Autonomous Systems, pp. 1508-1515.) underline the importance of teamwork and of the application of co-operating KBs. Local and global knowledge of autonomous systems are working in teams. There is a distributed, real-time, multi-user KB to facilitate teamwork in flexible manufacturing environments resulting in co-operating knowledge bases. Every local $\mathrm{KB}$ is an object structure with demons, multi-user and real-time access. Each local $\mathrm{KB}$ has an active component which asynchronously passes changed data to interested application processes. If several autonomous systems co-operate to perform a certain task, the local KBs of these systems form a team to provide the 
knowledge needed by the autonomous systems. The local KBs co-operate by means of selective updating and location-transparent access to the global knowledge. The local KBs are responsible for task planning, motion control, path planning and manipulation control.

N. Koyachi, T. Arai et al. in [36] (Geometric Design of Hexapod with Integrated Limb Mechanism of Leg and Arm, Vol. 3. pp. 291-296.) and in [35] (pp. 139-144.) discuss analysis and design problems of MELMANTIS, an autonomous hexapod walking robot.

A. Mahajan and F Figuerola in [4] (Four-Legged, Intelligent Mobile Autonomous Robot, Vol. 13, No. 1.pp. 51-61.) deal with application of intelligent sensors to design and implement a four-legged walking robot. The robot has intelligent sensing and decision-making capabilities. There are multiple sensors with embedded incremental learning is a new approach towards environmental perception and reaction. The sensors continuously monitor the environment as well as their own parameters. The name of the four-legged robot is FLIMAR. Each of the legs have three degree of freedom, i.e. there are 12 motors to move the robot. It responds to light, sound and touch in different ways, based on the environmental conditions. There are different degrees of intelligence at different levels of the $\mathrm{OO}$ architecture.

\subsection{Control Application}

N. Miyake, M. Kametani and H. Sakairi in [32] (Hardware and Software Architecture for Intelligent Robot Control - An Approach to Dual Control, pp. 2146-2151.) deal with increased human involvement. The intelligence of human should be incorporated into the robot controller by means of a dual control architecture having specific software and hardware tools, however the application of specific hardware tools is against recent control trends, where more and more burden is pushed over to the PCs.

Two layers are defined, where the upper one is analogous to the human cerebrum (symbolic data processing regarding the robot's global conditions and of the robot environment), the lower one is closed to the traditional controller. An intelligent system processor and motion control processor are working in synergy.

\section{CONCLUSIONS, FURTHER TRENDS}

It is clear that the application of computational, multi-agent and AI techniques and tools to design and operate robotic systems already has some really positive results world-wide, however most of these results are known and applied by the academic community, as industry needs more time to be convinced to use most up-to-date techniques. And if we want to be very honest, some of the results reported in literature are experimental or prototype solutions, thus real industrial 
applications are a little behind. It is not a problem, as several years ago the same could be (and was) stated about CAD and CAM techniques and tools, and now these are widely used in industry.

And as there are „too many" different techniques, such as genetic algorithms, KB, Fuzzy, ANN systems, Petri nets, holonic systems, concurrent engineering, multi-agent techniques, etc. which all can be combined with each other and with other AI tools as pattern recognition in the fields of vision, voice and tactile applications, there are lots of different new possibilities for future research and development tasks to be solved.

There is a trend to be more and more multidisciplinary to combine engineering tools and knowledge not only with computer technique tools and knowledge but with psychological results as well, which makes the future possibilities even more challenging and interesting.

H. Inoue in [16] (Whither Robotics: Key Issues, Approaches and Applications, pp. 9-14.) has some new ideas for the future suggesting rather new research issues for robotics, such as:

- physical understanding of dexterity

- real world understanding through tightly-coupled sensing and action

- human-robot co-operative systems

- biologically inspired autonomous systems

A triple shifting of research is predicted :

- from separated vision/control/intelligence/design to perception-action coupling in all levels

- from the motion of arms, legs, fingers, etc. to whole-body motion of robots

- from technical research to psychological and biological research.

Researchers are working already on some parts of these suggestions, some other parts are in the understanding and comprehension phase, and some of them will probably turn out to be false, what is natural in the case of real, new research directions.

On the other hand the author is convinced that several tangible results of the research are used already and will be used soon in industry, as for example the world-wide IMS project has achieved great improvements in holonic and lean manufacturing, in concurrent engineering and in several other robotics related topics. These, and other results in autonomous, mobile and other specific robotics are used in mining, space and underwater applications, etc. Finally we can state that in spite of all difficulties more and more intelligent, multi-agent and other systems are used for simulation, design and control of robotic systems in manufacturing, assembly and other applications.

\section{REFERENCES}

[1] Robotica, Cambridge University Press, UK

[2] Data and Knowledge Engineering, North Holland (Elsevier), Netherlands. 
[3] Machine Learning, Kluwer Academic Publishers, Netherlands.

[4] Robotics and Computer Integrated Manufacturing, Pergamon, Elsevier.

[5] Artificial Intelligence, Elsevier, Amsterdam.

[6] Computers in Industry, Elsevier, Amsterdam.

[7] Engineering Applications of AI, Elsevier, Oxford, UK, Pergamon Press.

[8] Artificial Intelligence in Engineering, Elsevier, Oxford, UK, Pergamon Press.

[9] Applied Intelligence, Kluwer Academic Publishers, Netherlands.

[10] Journal of Intelligent Systems, Freund Publishing House, UK.

[11] International Journal of Intelligent Mechatronics, Design and Production, Middle East Technical University, Ankara, Turkey.

[12] Journal of Artificial Intelligence Research, AI Access Foundation and Morgan Kaufmann.

[13] Proc. of IROS'92 (IEEE/RSJ International Conference on Intelligent Robots and Systems), Raleigh, North Carolina, USA, July 7-10, 1992.

[14] Haidegger, G and Kopácsi, S.:Telepresence with Remote Vision in Robotic Applications, Proc. of the International Workshop on Robotics in the AlpeAdria-Danube Region, Budapest, Hungary, June 10-13, 1996, pp. 557-562.

[15] Integration of Robots into CIM (Ed. by R. Bernhardt, R. Dillman, K Hörmann and K. Thiery), Chapmann \& Hall, 1992.

[16] Proc. of IROS'96 (IEEE/RSJ International Conference on Intelligent Robots and Systems), Osaka, Japan, Nov. 4-8, 1996.

[17] Artificial Intelligence in Design, ed. D.T. Pham, Springer-Verlag, 1991.

[18] Proceedings of the first World Congress on Intelligent Manufacturing, Processes and Systems, University of Puerto Rico, Mayagüez, Puerto Rico, ed. V. Milacic, Febr. 1995.

[19] Artificial Intelligence Implications for CIM, ed. A. Kusiak, IFS (Publications) and Springer-Verlag, 1988.

[20] G.L. Kovács, I. Mezgár and S. Kopácsi: "Concurrent Design of Automated Manufacturing Systems Using Knowledge Processing Technology", Computers in Industry, 17, pp. 257-267, Elsevier, 1991.

[21] G.L. Kovács and I. Mezgár: "Expert Systems for Manufacturing Cell Simulation and Design", in Engineering Applications of Artif. Intelligence, ed. M. G. Rodd, vol. 4, no. 6, pp. 417-421, Pergamon Press, 1991.

[22] G.L. Kovács, S. Kopácsi and I. Mezgár: "A PROLOG Based Manufacturing Cell Design System"; Practical Application of Prolog Conf., Abstracts of the Poster Sessi, April 1992, London, The Institution of Civil Engineers, pp. 3-4.

[23] G.L. Kovács and S Kopácsi: "Cooperative Agents for the Simulation of FMC and FMS", TIMS/ORSA Conference, Orlando, USA, April 1992.

[24] Kovács, G.L., Létray, Z.: Expert System for Work Cell Simulation and Design in PROLOG Language, the COOPERATOR; COMPCONTROL'89, Bratislava, CSSR, Sept. 12-14. 1989, section: 2.4

[25] Kovács, G.L.: Expert System for Manufacturing Cell Design; IFAC 6th Symposium on Automation in Mining, Mineral and Metalprocessing 
(MMM), Buenos-Aires, Argentina, 1989, Sept 4-8, Preprints: Volume 2. Session: Control 1, TS 14.1. pp. 324-329.

[26] Kovács, G.L., Létray, Z., Kopácsi, S. : Application of Cooperative Agents for Simulation of Flexible Manufacturing Systems (IFIP Intern. Workshop on Open Distributed Processing, 8-11. Oct. 1991), IFIP Transactions, C-1, North-Holland, 1992, Ed. J. De Meer, V. Heimer, R. Roth, pp. 415-420.

[27] Molina, A., Mezgár, I., Kovács, G: Cooperative Hybrid Expert Systems for Concurrent Design of FMS; Preprints of the IFAC Workshop on Int. Manuf. Syst., Dearborn, Michigan, USA. Oct 1-2, 1992, Ed. R. P. Judd and N. A. Kheir, Pergamon Press, pp. 51-56.

[28] Artificial Intelligence, Manufacturing Theory and Practice, ed. S.T. Kumara, A.S. Soyster and R.L. Kasyap, Industrial Eng. and Managm. Press, IIE, 1988.

[29]Realising CIM's Industrial Potential, IOS Press, Esprit CIM-Europe, 1993, Ed. Kooij, MacConnail and Bastos.

[30] Proc. of the 11th ISPE/IEE/IFAC International Conference on CAD/CAM. Robotics and Factories of the Future, Pereira, Colombia, August 28-30, 1995.

[31] Proc. of IROS'93 (IEEE/RSJ International Conference on Intelligent Robots and Systems), Jokohama, Japan, July 26-30, 1993

[32] Proc. of IROS'94 (IEEE/RSJ International Conference on Intelligent Robots and Systems), Munich, Germany, Sept. 12-16, 1994.

[33] N.J. Nilsson, P.R. Cohen and J. Rosenschein: Intelligent Communication Agents, Technical report, SRI International, 1987.

[34] Proc. of the Second World Congress on Intelligent Manufacturing Processes \& Systems, Budapest, Hungary, June 10-13, 1997.

[35] Proc. of the Japan-USA Symposium on Flexible Automation, Boston, USA, July 7-10, 1996.

[36] Proc. of IROS'95 (IEEE/RSJ International Conference on Intelligent Robots and Systems), Pittsburgh, USA, August 5-9, 1995.

\section{BIOGRAPHY}

George L. KOVACS got his diploma (Masters) of Electrical Engineering, $\mathrm{PhD}$, and Habil. (Prof.) degrees from the Technical University of Budapest (TUB) in 1966, 1976 and 1995 respectively. He got the Candidate of Sci. and D.Sc. degrees from the Hungarian Academy of Sciences (HAS) in 1978 and 1997 respectively. He has been working in the Computer and Automation Research Institute of the HAS since 1966. Recently he is the head of the CIM Research Laboratory. He has been serving for longer periods in the USA, Russia, Germany, Mexico and Italy as visiting researcher/professor. He published more than 150 papers in conference proceedings and in scientific journals on $\mathrm{CAD} / \mathrm{CAM} / \mathrm{CIM}$, intelligent manufacturing, etc. He is a member of IEEE, and member in several IFAC, IFIP and other committees, as well as in different Hungarian scientific boards and committees. 